\title{
Omega-3 Polyunsaturated Fatty Acid Biomarkers and Risk of Type 2 Diabetes, Cardiovascular Disease, Cancer, and Mortality: A Systematic Review and Meta-analysis
}

\author{
Hong Jiang \\ Xian Jiaotong University: Xi'an Jiaotong University \\ Lina Wang \\ Xian Jiaotong University: Xi'an Jiaotong University \\ Duolao Wang
}

Liverpool School of Tropical Medicine

Ni Yan

Xian Jiaotong University: Xi'an Jiaotong University

Chao Li

Xian Jiaotong University: Xi'an Jiaotong University

Min Wu

Xian Jiaotong University: Xi'an Jiaotong University

Jing Lin

Xian Jiaotong University: Xi'an Jiaotong University

Wanru Jia

Xian Jiaotong University: Xi'an Jiaotong University

Xi Liu

Xian Jiaotong University: Xi'an Jiaotong University

Jiaxin LV

Xian Jiaotong University: Xi'an Jiaotong University

Yan Liu

Xian Jiaotong University: Xi'an Jiaotong University

lema ( $\nabla$ male@mail.xjtu.edu.cn )

Xian Jiaotong University: Xi'an Jiaotong University https://orcid.org/0000-0001-7592-9779

\section{Original investigation}

Keywords: omega-3 polyunsaturated fatty acid biomarker, type 2 diabetes, cardiovascular disease, cancer, mortality, meta-analysis 
Posted Date: April 1st, 2021

DOI: https://doi.org/10.21203/rs.3.rs-362088/v1

License: (c) (i) This work is licensed under a Creative Commons Attribution 4.0 International License. Read Full License 


\section{Abstract}

Background: Considerable attention has focused on omega-3 polyunsaturated fatty acids (PUFA) role in protect against the development of cardiometabolic diseases, which has led to dietary recommendations to increase omega-3 fatty acid intake.

Methods: MEDLINE, EMBASE, ISI Web of Science, Cochrane Library, and reference lists were searched for articles from inception to May 2020. Random-effects model was used to estimate the pooled relative risk (RR) and 95\% confidence intervals (Cls) for the association of omega-3 PUFAs, including a-linoleic acid (ALA), eicosapentaenoic acid (EPA), docosapentaenoic acid (DPA), and docosahexaenoic acid (DHA), with risk of developing type 2 diabetes (T2D), cardiovascular disease (CVD), including coronary heart disease (CHD) and stroke, cancer, and mortality.

Results: 66 prospective studies comprised of 211,600 participants were identified. Individual omega-3 PUFAs showed divergent associations with the study outcomes of interest. An inverse association with risk of T2D was observed comparing extreme categories of ALA concentration (RR,0.91;95\%Cl,0.83-0.99), but not for the marine-origin omega-3 fatty acids biomarkers. The marine-origin omega-3 fatty acids biomarkers, but not ALA, were significantly associated with lower risks of total CVD, CHD, and overall mortality, with RRs ranging from 0.70 for DHA-CHD association to 0.85 for EPA-CHD association. Lower risk of colorectal cancer was observed at higher levels of DPA (RR,0.76;95\% Cl:0.59-0.98) and DHA (RR,0.80;95\% Cl:0.65-0.99). In dose-response analyses, inverse linear associations were observed between EPA, DPA, and DHA biomarkers and CVD or CHD risk, except for DHA-CVD association which showed a nonlinearity association.

Conclusion: Higher concentrations of marine-derived omega-3 PUFA biomarkers were associated with a significantly reduced risk of total CVD, CHD, certain types of cancer, and total mortality. Levels of ALA were inversely with a lower risk of T2D but not CVD-related outcomes. These data support the dietary recommendations advocating the role of omega-3 PUFAs in maintaining an overall lower risk of developing cardiovascular disease and premature deaths.

\section{Background}

Increasing polyunsaturated fatty acid (PUFA) consumption, especially seafood-derived omega-3 PUFAs, has been considered as a key component of prevention strategy in tackling the current epidemic of chronic disorders [1,2]. Dietary guidelines of the American Heart Association recommend a daily consumption of $250 \mathrm{mg}$ eicosapentaenoic acid (EPA) and docosahexaenoic acid (DHA) for decreasing the risk of cardiac deaths among individuals with and without pre-existing cardiovascular disease (CVD) [2]. Accumulating evidence from experimental studies has demonstrated that omega-3 PUFAs have multiple critical health benefits including inhibiting inflammation, regulating lipid metabolism, reducing arrhythmias, and improving endothelial function and insulin sensitivity [3,4]. Recently, the efficacy of marine-derived omega-3 PUFA supplementation for CVD risk reduction has been demonstrated in the 
Reduction of Cardiovascular Events with Icosapent Ethyl-Intervention Trial (REDUCE-IT) and the Vitamin D and Omega-3 Trial (VITAL) which consistently reported a beneficial effect of these fatty acids on reducing coronary heart disease (CHD) risk in the overall population or subgroups $[5,6]$. However, whether these effects can be extrapolated to general populations with lower habitual intake of omega-3 PUFAs or to other related health conditions is unclear.

Existing prospective cohort studies have examined associations of dietary omega-3 PUFAs with the incidence of major chronic diseases and mortality in free-living individuals, and findings of these studies were mixed $[7,8]$. Some studies showed that intake of plant-derived fatty acid, such as a-linolenic acid (ALA), was associated with decreased risk of T2D, whereas others have reported no such association [911]. The conflicting results might be related to variation in different population characteristics, different background diet, measurement errors of dietary assessments, as well as bioavailability of these fatty acids [12]. Meaningful amounts of omega-3 PUFAs could also be obtained from various fortified foods, making an accurate assessment of these fatty acids intake more challenging [13]. Biomarkers of omega3 PUFAs are valuable when evaluating the associations between the intake of these PUFAs and disease risk because they are free of reporting bias and other measurement errors intrinsic to questionnaire-based assessments $[14,15]$. Previous researches to date have focused mainly on the association with CVD risk and most suggested inverse associations [16-18]. Compared with the literature on CVD, less evidence exists regarding the associations of omega-3 PUFA fatty acid biomarkers and other chronic conditions, such as T2D, cancer or mortality. In addition, much existing evidence surrounds EPA and DHA, with relatively little evidence generated for the association between other omega-3 PUFAs, such as ALA or docosapentaenoic acid (DPA).

We therefore conducted a comprehensive dose-response meta-analysis of data from prospective studies to evaluate associations of omega-3 PUFA biomarkers with incident of T2D, total CVD, CHD, stroke, cancer and mortality.

\section{Methods}

\section{Search strategy and selection criteria}

The study was reported according to the preferred reporting items for systematic reviews and metaanalyses (PRISMA) guidelines (Additional file 1: Appendix S1) [19]. PubMed, EMBASE, Web of Science, and Cochrane Library were searched for relevant published articles from inception to May 2020. Three investigators (HJ, LNW, and MW) independently searched and further assessed the eligibility of all identified citations. Further details on the literature search, data extraction is described in Additional file 1: Appendix S2-S3.

\section{Eligibility criteria}


Studies were eligible for inclusion if they met the following criteria: (1) study design was prospective (prospective cohort, nested case-control, and case-cohort study) with a follow-up more than one year; (2) the exposures of interest were omega-3 PUFA concentration (ALA, and total or individual marine-derived omega-3 fatty acids) in any type of tissue (circulating blood or adipose tissue); (3) the endpoints of interest included incident T2D, total CVD, CHD, stroke, cancer, all-cause mortality, and cause-specific mortality; (4) the risk estimate with corresponding 95\% confidence intervals (Cls) or standard error was presented.

\section{Quality assessment}

Study quality was scrutinized by the same authors following the validated Newcastle-Ottawa scale, which awards 0-9 points and incorporates information on selection, comparability, and outcome assessment. We defined studies as low, moderate, and high quality for those scored 0-3, 4-6, and 7-9, respectively [20].

\section{Data synthesis and statistical analysis}

Methods previously described were used to derive estimates of associations corresponding to the comparison between the top and bottom thirds of omega-3 PUFA distributions [21]. When studies used multiple measures as biomarker, the overall risk estimate was based on different duration of intake reflection according to the following list: adipose tissue, erythrocyte phospholipids, plasma phospholipids, total plasma or serum, and cholesterol esters. For each included study, the most fully adjusted estimates of rate, hazard, or odds ratios from prospective studies were all valid estimates of the RR. Studies that reported results by sex or other subgroups separately were pooled to derive a single effect size for the study. When CHD and stroke outcomes were separately provided in the same study, we did not combine it to obtain total CVD risk estimates, and therefore, the CVD analysis only considered studies that examined total CVD incidence.

Random-effects model was used to calculate summary RR and $95 \%$ Cls. Heterogeneity across study effects was assessed using $Q$ test and $R$ statistic. When statistically significant heterogeneity was detected, meta-regression analyses were performed to explore sources of heterogeneity, including study design, sex, geographical location, duration of follow-up, assessment method, biological sample type, number of cases, and study quality.

Dose-response analyses were assessed using the method proposed by Greenland and Longnecker to calculate study linear trends and $95 \% \mathrm{Cls}$ from the natural logs of the RRs and Cls across categories of omega-3 PUFA exposure [22]. The dose-response outcomes were limited to studies that reported circulating omega-3 PUFA due to the small number of included studies with other exposures. Sensitivity analyses were performed to further evaluate the robustness of the conclusions. Potential publication bias was examined by Begg's and Egger's tests, as well as the trim and fill method. All analyses were 
performed using Stata, version 10.2 (Stata Corp, Texas), and $P<0.05$ was considered statistically significant. Details of the statistical methods is available in Additional file 1: Appendix S2.

\section{Results}

\section{Study characteristics}

Figure 1 summarizes the literature search and selection process. We identified 17,339 citations in the primary search, of which 162 were retrieved for full text evaluation after the initial screening of abstracts and titles. 12 studies were identified through manual examination of reference lists. Overall, a total of 66 analyses reported in 64 articles were included in our main analysis (Additional file 1: Appendix S4-9).

The included studies comprised of 25 prospective cohort studies, nine case-cohort studies, one nested case-cohort study, and 30 nested case-control studies. Twenty-five studies were conducted in Europe, 28 in the United States, seven in Asia, and five in Australia. Mean age of participants in the individual studies ranged from 41.1 to 80.5 years. For the measurements of omega-3 PUFA levels, 51 studies used gas chromatography (GC) analytic approach, 13 used gas-liquid chromatography, and one used GC-tandem mass spectrometry.

The Additional file 2: Appendix S1 shows the mean (standard deviation [SD]) proportion of each objective omega-3 PUFA relative to the total fatty acid contents in blood compartments or tissues. Fifty-eight studies were deemed to be of high quality, and the others judged as having a moderate quality (Additional file 1: Appendix S10-S11).

\section{Omega-3 PUFA biomarkers and type 2 diabetes}

Eleven studies comprised of 15,595 T2D incidence and 53,642 participants investigated the relationship between the concentrations of omega-3 PUFA biomarkers and T2D risk. Compared with participants in the lowest tertile, those in the highest tertile of ALA levels had a lower risk of T2D (RR,0.91;95\%Cl,0.83$0.99 ; P_{\text {heterogeneity }}=0.26$; Fig. 2 and Additional file 2: Appendix S2). No significant associations were found for levels of EPA (RR,0.87;95\% Cl: 0.75-1.00; $P_{\text {heterogeneity }}<0.001 ;$ Fig. 2), DPA (RR,0.87;95\%Cl,0.76$1.00 ; P_{\text {heterogeneity }}=0.001 ;$ Fig. 2$), \mathrm{DHA}\left(\mathrm{RR}, 0.92 ; 95 \% \mathrm{Cl}, 0.78-1.10 ; P_{\text {heterogeneity }}=0.001 ;\right.$ Fig. 2$)$ or the sum of $\mathrm{EPA}+\mathrm{DPA}+\mathrm{DHA}\left(\mathrm{RR}, 0.81 ; 95 \% \mathrm{Cl}, 0.60-1.09 ; P_{\text {heterogeneity }}=0.15 ; \mathrm{Fig} .2\right)$ with T2D risk (Additional file 2: Appendix S3-6 and Additional file 1: Appendix S12). The result of subgroup analyses did not substantially alter the association between level of omega-3 PUFA biomarkers and T2D risk. In dose-response analyses, a linear association was found between ALA biomarker and T2D risk (Additional file 2: Appendix S7). The RR of T2D for each 1-SD increase in concentrations of ALA was $0.91(95 \% \mathrm{Cl}, 0.83-$ 0.99).

\section{Omega-3 PUFA biomarkers and CVD}

Association of omega-3 PUFA levels with total CVD was assessed in 12 studies, which included a total of 5,503 cases among 35,581 participants. When comparing the extreme tertiles, the risk of total CVD was 
significantly lower by $19 \%$ for EPA (RR,0.81;95\%Cl,0.72-0.91; $P_{\text {heterogeneity }}=0.30 ;$ Fig. 2$), 23 \%$ for DPA $\left(\mathrm{RR}, 0.77 ; 95 \% \mathrm{Cl}, 0.69,0.85 ; P_{\text {heterogeneity }}=0.94\right.$; Fig. 2$), 25 \%$ for DHA (RR,0.75;95\%Cl, $0.65-$

$0.87 ; P_{\text {heterogeneity }}=0.01$; Fig. 2$)$, and by $55 \%$ for the sum of EPA + DPA + DHA (RR, $0.45 ; 95 \% \mathrm{Cl}, 0.27-$ $0.74 ; P_{\text {heterogeneity }}=0.15 ;$ Fig. 2) (Additional file 3: Appendix S1-S4 and Additional file 1: Appendix S13). No association was observed between ALA and risk of CVD when comparing the highest with lowest categories (RR,1.09;95\% Cl,0.99-1.21; $P_{\text {heterogeneity }}=0.65$; Fig. 2 and Additional file 3: Appendix S5). Results from the dose-response analyses showed a significant linear decrease in the risk of CVD of individuals with increasing values of circulating EPA and DPA concentration, and per 1-SD increment was associated with $9 \%(R R, 0.91 ; 95 \% \mathrm{Cl}, 0.86-0.96)$ and $11 \%(\mathrm{RR}, 0.89 ; 95 \% \mathrm{Cl}, 0.85-0.93)$ lower risk of $\mathrm{CVD}$, respectively (Fig. 3). A potential nonlinear dose-response curve was detected for DHA-CVD association in that the CVD risk did not decrease until the DHA levels exceeded about $2 \%\left(P_{\text {non-linearity }}=0.02\right.$; Fig. 3$)$.

\section{Omega-3 PUFA biomarkers and CHD}

The association between omega-3 PUFA biomarker levels and CHD was evaluated in 13 studies, which consisted of 7,626 cases and 27,814 participants. The overall effect estimates of CHD comparing the top tertile compared with bottom tertile was 0.98 for $\mathrm{ALA}\left(95 \% \mathrm{Cl}, 0.95-1.02 ; P_{\text {heterogeneity }}=0.89\right.$; Fig. 2$), 0.85$ for EPA ( $95 \% \mathrm{Cl}, 0.77-0.95 ; P_{\text {heterogeneity }}=0.41$; Fig. 2$), 0.83$ for DPA $\left(95 \% \mathrm{Cl}, 0.76-0.92 ; P_{\text {heterogeneity }}=0.84 ;\right.$ Fig. 2$)$, 0.70 for DHA $\left(95 \% \mathrm{Cl}, 0.58-0.84 ; P_{\text {heterogeneity }}=0.02\right)$; Fig. 2 , and 0.67 for the sum of EPA + DPA + DHA $\left(95 \% \mathrm{Cl}, 0.47-0.96 ; P_{\text {heterogeneity }}=0.34 ;\right.$ Fig. 2) (Additional file 4: Appendix S1-S5 and Additional file 1: Appendix S13). For the dose-response analyses, a linear association was observed for marine-derived omega-3 PUFA biomarkers and risk of CHD. For every 1-SD increase in levels of EPA, DPA, and DHA in circulating, the RR: of $\mathrm{CHD}$ decreased by $8 \%(\mathrm{RR}, 0.92 ; 95 \% \mathrm{Cl}, 0.88-0.97), 17 \%(\mathrm{RR}, 0.83 ; 95 \% \mathrm{Cl}, 0.74-1.06)$, and $16 \%$ (RR:0.84;95\% Cl,0.77-0.93), respectively (Fig. 3).

\section{Omega-3 PUFA biomarkers and stroke}

Twelve studies provided information on omega-3 PUFA levels and the subsequent risk of stroke, including a total of 7,036 events in 77,163 participants. The pooled estimate indicated that high DHA status was associated with a lower risk of stroke (RR,0.84;95\% Cl,0.72-0.99; $P_{\text {heterogeneity }}=0.03$; Fig. 2$)$, while there was no significant association for biomarkers of ALA, EPA, DPA, or the sum of EPA + DPA + DHA (; Fig. 2, Additional file 5: Appendix S1-S5 and Additional file 1: Appendix S14). A linear relation was noted between DHA biomarker and stroke in the dose-response analysis (Additional file 5: Appendix S6), and the RR: was 0.91 (95\% Cl,0.83-0.99) for each 1-SD increment of DHA concentration in circulating.

\section{Omega-3 PUFA biomarkers and cancer}

Twenty-one studies were included in the analysis of omega-3 PUFA biomarker status and colorectal, breast, or prostate cancers. For colorectal cancer $(n=3)$, in comparison with the lowest category, the highest level category of DPA and DHA were associated with $24 \%(\mathrm{RR}, 0.76 ; 95 \% \mathrm{Cl}, 0.59-$ $0.98 ; P_{\text {heterogeneity }}=0.87 ;$ Fig. 2$)$ and $20 \%\left(\mathrm{RR}, 0.80 ; 95 \% \mathrm{Cl}, 0.65-0.99 ; P_{\text {heterogeneity }}=0.56 ;\right.$ Fig. 2$)$ reduced risk of colorectal cancer, respectively (Additional file 6: Appendix S1-S2). ALA and EPA biomarker had a non- 
significant association with incident colorectal cancer (Additional file 6: Appendix S3-S4). No association was observed between ALA, EPA, DPA, and DHA concentrations and incidence of breast cancer $(n=9)$ or prostate cancer ( $n=9)$ (Fig. 2 and Additional file 6: Appendix S5-S12 and Additional file 1: Appendix S15).

\section{Omega-3 PUFA biomarkers and total mortality}

Eight studies investigated the relation of omega-3 PUFA biomarker levels with mortality with a total of 7,558 deaths from 23,484 participants. Pooled RR for the comparison of extreme tertiles was 0.80 for EPA $\left(95 \% \mathrm{Cl}, 0.71-0.90 ; P_{\text {heterogeneity }}=0.02\right.$; Fig. 2$), 0.81$ for DPA $\left(95 \% \mathrm{Cl}, 0.73-0.90 ; P_{\text {heterogeneity }}=0.59 ;\right.$ Fig. 2$)$, and 0.84 for DHA (95\% Cl,0.76-0.94; $P_{\text {heterogeneity }}=0.07$; Fig. 2) (Additional file 7: Appendix S1-S3 and Additional file 1: Appendix S16). Nonsignificant association was observed for ALA biomarker (RR,1.00;95\%Cl,0.93-1.08; $P_{\text {heterogeneity }}=0.64$; ; Fig. 2 and Additional file 7: Appendix S4).

\section{Sensitivity analysis and publication bias}

In sensitivity analyses omitting one study at a time from each analysis, the combined estimate did not substantially change for most omega-3 PUFA biomarkers, except for the studies that evaluated the association between DHA level and prostate cancer: the pooled RR $(95 \% \mathrm{Cl})$ was strengthened to 1.14 (95\% Cl,1.00-1.30) when the study by Chavarro et al. was removed [23]. No indication of substantial publication bias was found for most outcomes with either Egger's test or Begg's test $(P>0.05$ for both tests; Additional file 7: Appendix S5-S7).

\section{Discussion}

This comprehensive meta-analysis demonstrated robust inverse associations between marine-derived omega-3 PUFA in circulation or adipose tissue and lower risk of total CVD, CHD and overall mortality. Furthermore, a high level of DPA and DHA were inversely associated with colorectal cancer risk. In contrast, the associations between ALA and disease outcomes are less clear except an inverse association for T2D. The results largely persisted in dose-response meta-analyses or sub-group analyses. These findings therefore suggest that omega-3 PUFAs have important implications in chronic diseases prevention.

Results from large-scale randomized controlled trials (RCTs) that investigated the effects of omega-3 PUFA supplementation on composite cardiovascular end points have shown conflicting results. Data from a previous meta-analysis of 10 RCTs (77,917 patients with existing cardiovascular conditions) reported no significant adverse or beneficial effects of omega-3 fatty acid supplements on CHD and major vascular events risk for a mean of 4.4 years treatment [24]. However, two recent randomized trials, which were conducted among individuals who were free of pre-existing CVD, suggested cardiovascular benefits by omega-3 fatty acid supplementation. According to the REDUCE-IT report, supplementation with pure EPA at $4 \mathrm{~g} /$ day decreased the risk of ischemic events by $19-30 \%$ during a median of $4.9 \mathrm{y}$ among 8,179 patients with elevated CVD risk or diabetes [5]. Results from the VITAL also showed a significant $39 \%$ reduction in myocardial infarction (MI) risk by $1 \mathrm{~g} /$ day EPA + DHA supplementation over a 
median 5.3 years period [6]. In agreement with the results of these two trials, we also found a significant, lower risk of developing CHD and CVD with higher levels of EPA, DPA, and DHA biomarkers among largely healthy individuals, which lends further support for the role of these nutrients in the primary prevention of CHD and CVD.

In contrast with cardiometabolic diseases, no RCT has yet been performed to explore the effects of omega-3 PUFA supplementation on risk of T2D incidence. Existing trials that evaluated the effect of fish oil supplementation and risk markers of T2D yielded mixed results. In a 12-week RCT, Thota et al. detected that marine-derived omega-3 PUFA supplementation produced a significant improvement in insulin resistance among participants at high risk of developing diabetes, impaired fasting glucose or glucose tolerance at baseline [25]. However, Giacco et al. did not observe significant effect on insulin action, $\beta$-cell function, or glucose tolerance with omega-3 PUFA supplementations in participants without T2D during a 3 months intervention period [26]. A number of studies that specifically evaluated fish intake (the main source of EPA, DPA, and DHA) and T2D risk have been conducted over the past several decades and few reported protective association $[27,28]$. The present study did not find evidence of an association between individual marine-derived omega-3 PUFA biomarkers and the risk of T2D, except for plant-derived ALA, for which an inverse trend was observed. The underlying mechanisms by which ALA may lower T2D risk may be partly due to the improvement in insulin sensitivity. It is likely that ALA could modulate nuclear receptors, such as peroxisome proliferator activated receptor, to improve insulin-like growth factor-I secretion from hepatocytes, which might help enhance whole-body insulin sensitivity and modulate carbohydrate metabolism. Moreover, the favorable effect of ALA to T2D might be also attributed to stimulating insulin secretion [29,30]. This hypothesis was supported by the evidence from in vivo and ex vivo studies, in which ALA was capable of stimulating glucose-dependent insulin secretion from pancreatic islets both directly and indirectly through mediating glucagon-like peptide 1 [31]. It is important to mention that circulating or adipose tissue ALA biomarker may not reflect ALA intake as dietary source of ALA was extensively oxidized after ingestion and absorption. In addition, it could convert to EPA and DHA after consumption and absorption, though the proportion is small [32, 33].

With regard to cancer, large intervention trials exploring omega-3 PUFA supplementation in the primary prevention of cancer are scarce. In the VITAL trial, omega-3 PUFA supplementation showed no effect on the incidence of cancer or death from cancer. ${ }^{6}$ However, these results should be interpreted with caution as the number of cases was small in the trial which made it difficult to draw small to modest effects. Observational studies that evaluated the associations of fish and omega-3 PUFA consumption with breast cancer, prostate cancer, or colorectal cancer did not yield consistent findings [34-36]. Results from our study indicated that a higher level of certain marine-derived omega-3 fatty acids was associated with a significant decreased risk of colorectal cancer. However, only a limited number of studies were conducted in this regard and further studies are warranted to substantiate the relationship between omega-3 PUFAs and cancer.

\section{Limitations of study}


Several potential limitations should be considered when interpreting the results. First, fatty acid biomarker levels were measured only once at baseline and changes of fatty acid levels over time were not accounted for. However, a recent analysis suggested that the omega-3 PUFA concentrations, with the exceptions of DPA, in serum cholesteryl ester, triglyceride and phospholipid fractions remained fairly stable in 8-10 years [37]. Second, although most included studies in our analysis adjusted for multiple major risk factors, such as sociodemographic, lifestyle, clinical, and other dietary risk factors, we cannot exclude the impact of residual and unmeasured confounding on the observed associations [38]. In particular, participants with high omega-3 PUFA status might be more likely to adhere to a healthier dietary pattern or have a higher socioeconomic status, which might distort the true associations. Third, variation in fatty acids metabolism and de novo lipogenesis between individuals and between populations may introduce extraneous heterogeneity to the current analysis. In the era of precision medicine, further studies are needed to incorporate factors that account for individual variation in response to omega-3 PUFA intake and subsequent chronic disease risk. Finally, we cannot exclude the possibility of publication bias, although our trim-and-fill analyses suggested such a bias is likely to be small.

\section{Conclusion}

Our meta-analysis of existing prospective evidence indicated that the marine-derived omega-3 PUFAs were associated with a lower risk of developing major chronic diseases, including CVD, CHD, certain types of cancer, and mortality, although associations for other disease outcomes were unclear. These findings further support the current recommendations of increasing intakes of marine-derived omega-3 PUFAs to facilitate the primary and secondary prevention of chronic conditions, especially CVD.

\section{Abbreviations}

ALA, a-linoleic acid; Cl, confidence interval; CHD, coronary heart disease; CVD, cardiovascular disease; DHA, docosahexaenoic acid; DPA, docosapentaenoic acid; EPA, eicosapentaenoic acid; GC, gas chromatography; PUFA, polyunsaturated fatty acid; RCT, randomized controlled trial; REDUCE-IT, the Reduction of Cardiovascular Events with Icosapent Ethyl-Intervention Trial; RR, relative risk; SD, standard deviation; T2D, type 2 diabetes; VITAL, the Vitamin D and Omega-3 Trial.

\section{Declarations}

\section{Ethics approval and consent to participate}

Not applicable.

\section{Consent for publication}

Not applicable. 


\section{Availability of data and materials}

The datasets used and/or analyzed during the current study are available from the corresponding author on reasonable request.

\section{Competing interests}

The authors declare that they have no competing interests.

\section{Funding}

This study was supported by grants from the National Natural Science Foundation of China (NSFC81473059; NSFC-81973025; NSFC-81202198); Nutrition Science Research Foundation of BY-HEALTH (TY0181101); the Natural Science Foundation of Shaanxi Province of China (2017JM8041); New-star Plan of Science and Technology of Shaanxi Province (2015LJXX-07); the Nutrition Research Foundation Fund of the Chinese Nutrition Society-DSM Special Research Foundation (CNSDSM 2016-041); and the Fundamental Research Funds for the Central Universities (qngz2016004; xzy032019008).

\section{Authors' contributions}

LM and DLW generated the idea for the study, formulated an analytical plan. All authors acquired, analyzed, or interpreted the data. HJ and LNW designed the search strategy, and $\mathrm{HJ}$ and LNW, and MW performed the literature search and screened studies for eligibility. HJ, YN, and MW extracted data. WRJ and $X L$ assessed the risk of bias. $\mathrm{HJ}, \mathrm{JXL}$, and $\mathrm{YL}$ performed data analysis. $\mathrm{HJ}, \mathrm{MW}, \mathrm{LM}$, and $\mathrm{CL}$ interpreted the data analysis and assessed the certainty of evidence. $\mathrm{HJ}$ drafted the manuscript and all other authors revised the manuscript. LM supervised the study. The corresponding author attests that all listed authors meet authorship criteria and that no others meeting the criteria have been omitted.

\section{Acknowledgements}

Not applicable

\section{References}

1. Chaddha A, Eagle KA. Cardiology patient page. Omega-3 fatty acids and heart health. Circulation. $2015 ; 132: 350-2$.

2. S. Department of health and human services and U.S. department of agriculture. 2015-2020 Dietary Guidelines for Americans. 8th Edition. December 2015. http://health.gov/dietaryguidelines/2015/guidelines/. Accessed 15 April 2020. 
3. Giordano E, Visioli F. Long-chain omega 3 fatty acids: molecular bases of potential antioxidant actions. Prostag Leukotr Ess. 2014; 90:1-4.

4. Elagizi A, Lavie C J, Marshall K, DiNicolantonio JJ, O'Keefe JH, Milani RV. Omega-3 polyunsaturated fatty acids and cardiovascular health: A comprehensive review. Prog Cardiovasc Dis. 2018; 61:76-85.

5. Bhatt DL, Steg PG, Miller M, Brinton EA, Jacobson TA, Ketchum SB, et al. REDUCE-IT Investigators. Effects of Icosapent Ethyl on total ischemic events: From REDUCE-IT. J Am Coll Cardiol. 2019; 73:2791-802.

6. Manson JE, Cook NR, Lee IM, Christen W, Bassuk SS, Mora S, et al. Marine n-3 fatty acids and prevention of cardiovascular disease and cancer. N Engl J Me. 2019; 380:23-32.

7. Zheng JS, Hu XJ, Zhao YM, Yang J, Li D. Intake of fish and marine n-3 polyunsaturated fatty acids and risk of breast cancer: meta-analysis of data from 21 independent prospective cohort studies. 2013; 346:f3706.

8. Medenwald D, Kluttig A, Lacruz ME, Schumann J. Serum dietary fatty acids and coronary heart disease risk-A nested case-control-study within the CARLA cohort. Nutr Metab Cardiovasc Dis. 2019; 29:152-8.

9. Brostow DP, Odegaard AO, Koh WP, Duval S, Gross MD, Yuan JM, et al. Omega-3 fatty acids and incident type 2 diabetes: the Singapore Chinese Health Study. Am J Clin Nutr. 2011; 94:520-6.

10. Dow C, Mangin M, Balkau B, Affret A, Boutron-Ruault MC, Clavel-Chapelon F, et al. Fatty acid consumption and incident type 2 diabetes: an 18-year follow-up in the female E3N (Etude Epidémiologique auprès des femmes de la Mutuelle Générale de l'Education Nationale) prospective cohort study. Br J Nutr. 2016; 116:1-9.

11. Djoussé L, Gaziano JM, Buring JE, Lee IM. Dietary omega-3 fatty acids and fish consumption and risk of type 2 diabetes. Am J Clin Nutr. 2011; 93:143-150.

12. Shim JS, Oh K, Kim HC. Dietary assessment methods in epidemiologic studies. Epidemiol Health. 2014; 36:e2014009.

13. Kris-Etherton PM, Harris WS, Appel LJ. Fish consumption, fish oil, omega-3 fatty acids, and cardiovascular disease. Circulation. 2002; 106:2747-57.

14. Arab L. Biomarkers of fat and fatty acid intake. J Nutr. 2003; 133:925S-32S.

15. Sun Y, Koh HW, Choi H, Koh WP, Yuan JM, Newman JW, et al. Plasma fatty acids, oxylipins, and risk of myocardial infarction: the Singapore Chinese Health Study. J Lipid Res. 2016; 57:1300-7.

16. Warensjo E, Sundstrom J, Vessby B, Cederholm T, Risérus U. Markers of dietary fat quality and fatty acid desaturation as predictors of total and cardiovascular mortality: a population-based prospective study. Am J Clin Nutr. 2008; 88:203-9.

17. Woodward M, Tunstall-Pedoe H, Batty GD, Tavendale R, Hu FB, Czernichow S. The prognostic value of adipose tissue fatty acids for incident cardiovascular disease: results from 3944 subjects in the Scottish Heart Health Extended Cohort Study. Eur Heart J. 2011; 32:1416-23. 
18. Mozaffarian D, Lemaitre RN, King IB, Song X, Huang H, Sacks FM, et al. Plasma phospholipid longchain $\omega-3$ fatty acids and total and cause-specific mortality in older adults: A cohort study. Ann Intern Med. 2013; 158:515-25.

19. Moher D, Shamseer L, Clarke M, Ghersi D, Liberati A, Petticrew M, et al. Preferred Reporting Items for Systematic Review and Meta-Analysis Protocols (PRISMA-P) 2015 statement. Syst Rev. 2015; 4:1.

20. Wells GA, Shea B, O'Connell D, Peterson J, Welch V, Losos M, et al. The Newcastle-Ottawa Scale (NOS) for assessing the quality of nonrandomised studies in meta-analyses. http://www.ohri.ca/programs/clinical_epidemiology/oxford.asp. Accessed 21 April 2020.

21. Danesh J, Collins R, Appleby P, Peto R. Association of fibrinogen, C-reactive protein, albumin, or leukocyte count with coronary heart disease: meta-analyses of prospective studies. JAMA. 1998; 279:1477-82.

22. Greenland S, Longnecker MP. Methods for trend estimation from summarized dose-response data, with applications to meta-analysis. Am J Epidemiol 1992; 135: 1301-9.

23. Chavarro JE, Stampfer J, LiH, Campos H, Kurth T, Ma J. A prospective study of polyunsaturated fatty acid levels in blood and prostate cancer risk. Cancer Epidemiol Biomarkers Prev. 2007; 16:1364-70.

24. Aung T, Halsey J, Kromhout D, Gerstein HC, Marchioli R, Tavazzi L, et al. Associations of omega-3 fatty acid supplement use with cardiovascular disease risks: meta-analysis of 10 trials involving 77917 individuals. JAMA Cardiol. 2018; 3:225-33.

25. Thota RN, Acharya SH, Garg ML. Curcumin and/or omega-3 polyunsaturated fatty acids supplementation reduces insulin resistance and blood lipids in individuals with high risk of type 2 diabetes: a randomised controlled trial. Lipids Health Dis. 2019; 18:31.

26. Giacco R, Cuomo V, Vessby B, Uusitupa M, Hermansen K, Meyer BJ, et al. Fish oil, insulin sensitivity, insulin secretion and glucose tolerance in healthy people: is there any effect of fish oil supplementation in relation to the type of background diet and habitual dietary intake of $n-6$ and n-3 fatty acids? Nutr Metab Cardiovasc Dis. 2007; 17:572-80.

27. Xun P, He K. Fish Consumption and incidence of diabetes: meta-analysis of data from 438,000 individuals in 12 independent prospective cohorts with an average 11-year follow-up. Diabetes Care. 2012; 35:930-8.

28. Wallin A, Di Giuseppe D, Orsini N, Åkesson A, Forouhi NG, Wolk A. Fish consumption and frying of fish in relation to type 2 diabetes incidence: a prospective cohort study of Swedish men. Eur J Nutr. 2017; 56:843-52.

29. Fang XL, Shu G, Zhang ZQ, Wang SB, Zhu XT, Gao P, et al. Roles of alpha-linolenic acid on igf-i secretion and gh/igf system gene expression in porcine primaryhepatocytes. Mol Biol Rep. 2012; 39:10987-96.

30. Clemmons DR. The relative roles of growth hormone and igf-1 in controlling insulin sensitivity. J Clin Invest. 2004; 113: 25-27.

31. Adachi T, Tanaka T, Takemoto K, Koshimizu TA, Hirasawa A, Tsujimoto G. Free fatty acids administered into the colon promote the secretion of glucagon-like peptide-1 and insulin. Biochem 
Biophys Res Commun. 2006; 340: 332-37.

32. Burdge GC, Calder PC. Conversion of alpha-linolenic acid to longer-chain polyunsaturated fatty acids in human adults. Reprod Nutr Dev. 2005; 45: 581-97.

33. Arterburn LM, Hall EB, Oken H. Distribution, interconversion, and dose response of n23 fatty acids in humans. Am J Clin Nutr. 2006; 83: 1467S-76S.

34. Sugawara Y, Kuriyama S, Kakizaki M, Nagai M, Ohmori-Matsuda K, Sone T, et al. Fish consumption and the risk of colorectal cancer: the Ohsaki Cohort Study. Br J Cancer. 2009; 101:849-54.

35. Pham TM, Fujino Y, Kubo T, Ide R, Tokui R, Mizoue T, et al. Fish intake and the risk of fatal prostate cancer: findings from a cohort study in Japan. Public Health Nutr. 2009; 12: 609-13.

36. Thiébaut $A C$, Chajès V, Gerber $M$, Boutron-Ruault MC, Joulin V, Lenoir $G$, et al. Dietary intakes of omega- 6 and omega-3 polyunsaturated fatty acids and the risk of breast cancer. Int J Cancer. 2009; 124:924-31.

37. Ohrvall M, Tengblad S, Gref CG, Salminen I, Aro A, Vessby B. Serum alpha tocopherol concentrations and cholesterol ester fatty acid composition in 70-year-old men reflect those 20 years earlier. Eur $\mathrm{J}$ Clin Nutr. 1996; 50:381-5.

38. Carter AR, Gill D, Davies NM, Taylor AE, Tillmann T, Vaucher J, et al. Understanding the consequences of education inequality on cardiovascular disease: Mendelian Randomisation Study. BMJ. 2019; 365:11855.

\section{Figures}


Study identified through literature search $(\mathrm{n}=17,339)$

Duplicate publications removed $(\mathrm{n}=2,475)$

Publication for screening on titles and abstract $(n=14,864)$

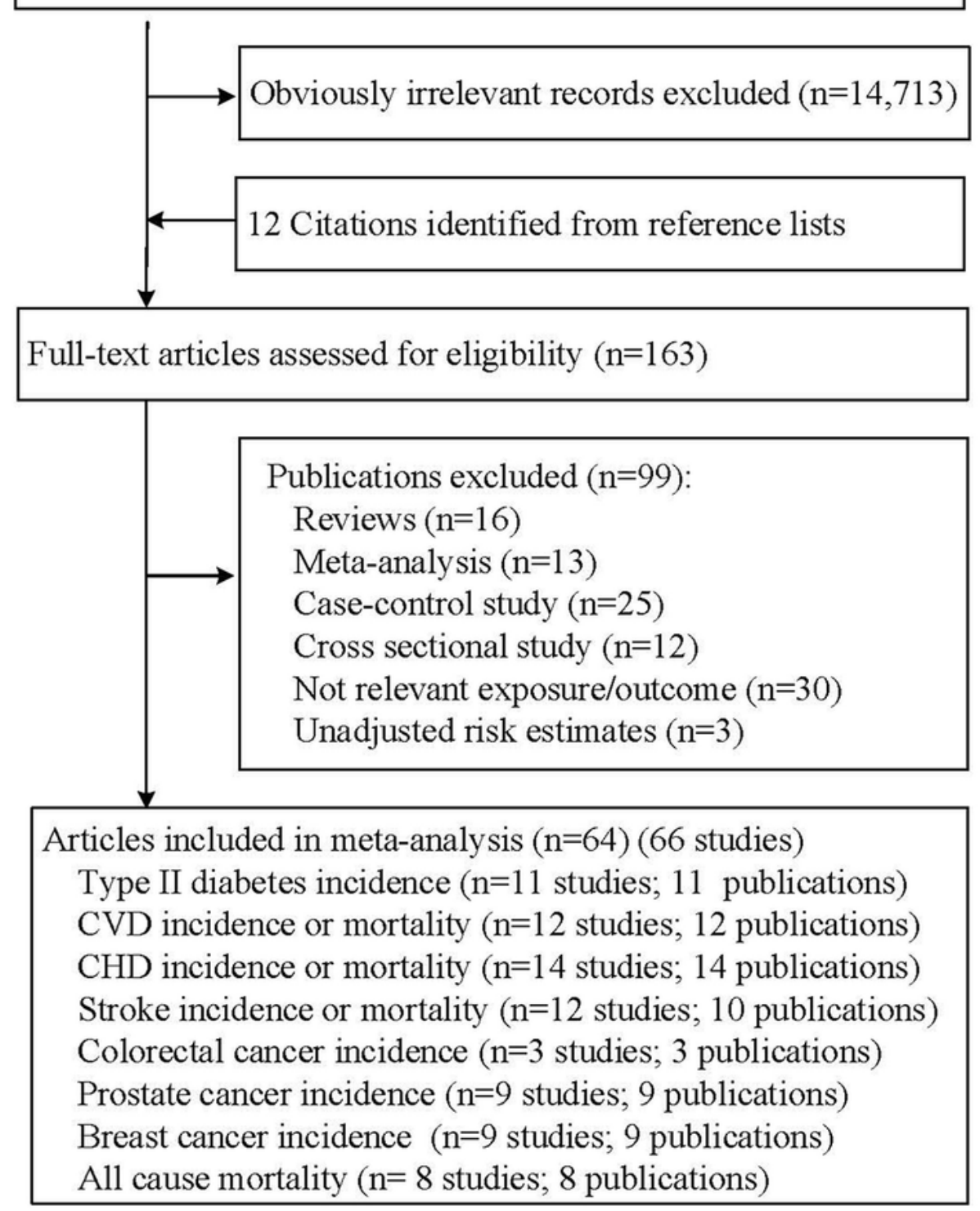

\section{Figure 1}

Flow chart of study selection CHD, coronary heart disease; CVD, cardiovascular disease; T2D, type 2 diabetes. 
Omega-3 fatty acid Studies, $n$ Events, $n$ Participants, $n$

ALA

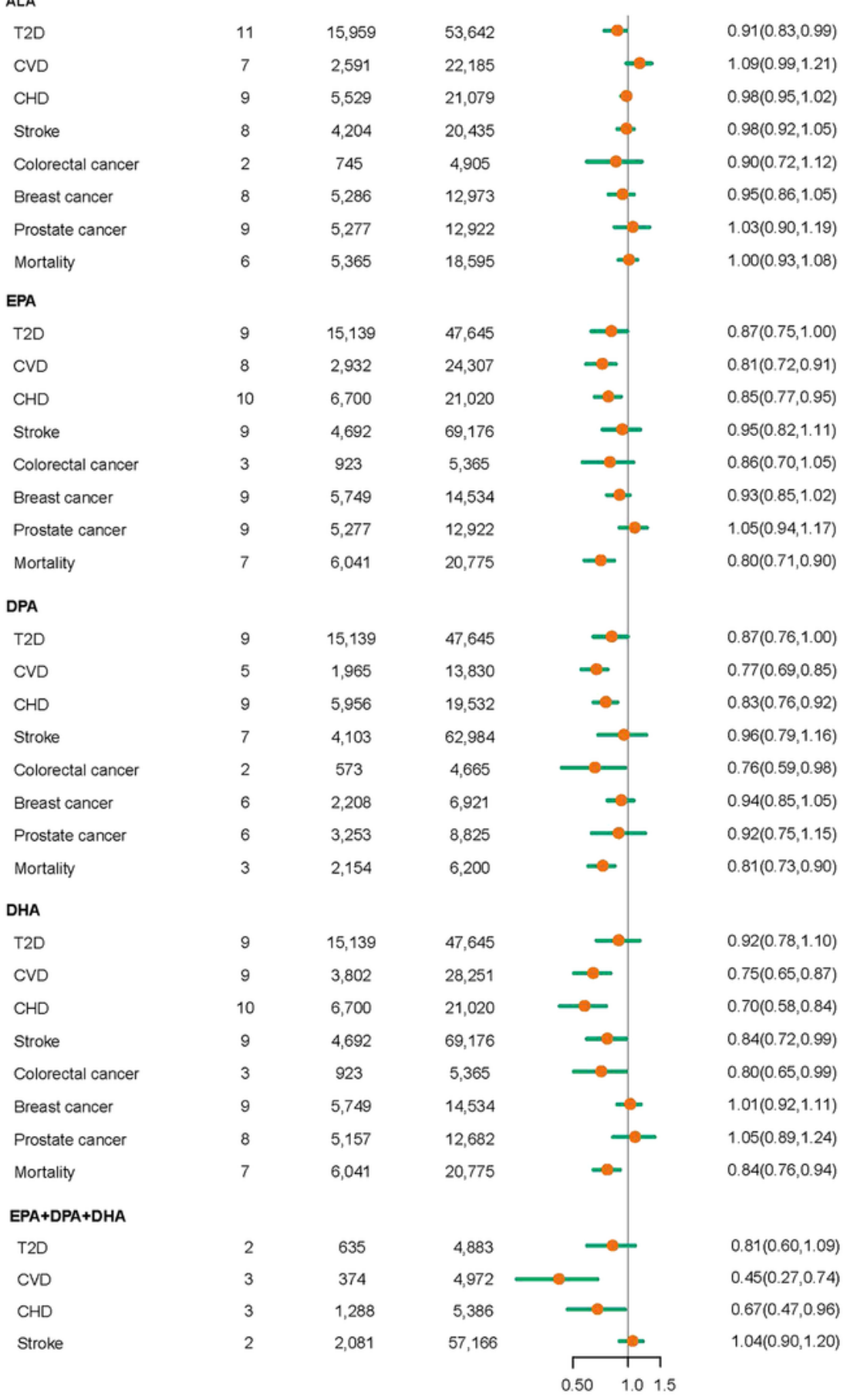

Relative risk (95\% CI)

\section{Figure 2}

Pooled relative risks (RRs) of T2D CVD, CHD, stroke, colorectal cancer, prostate cancer, and all-cause mortality comparing the highest with the lowest tertile of omega-3 fatty acids biomarkers. The size of the black squares indicates the relative weight of each estimate, horizontal lines indicate $95 \%$ confidence interval $(\mathrm{Cl})$, and diamonds indicate summary RR estimates with 95\% Cls. ALA, a-linolenic acid; CHD, 
coronary heart disease; $\mathrm{Cl}$, confidence interval; CVD, cardiovascular disease; DHA, docosahexaenoic acid; DPA, docosapentaenoic acid; EPA, eicosapentaenoic acid; RR, relative risk; T2D, type 2 diabetes.
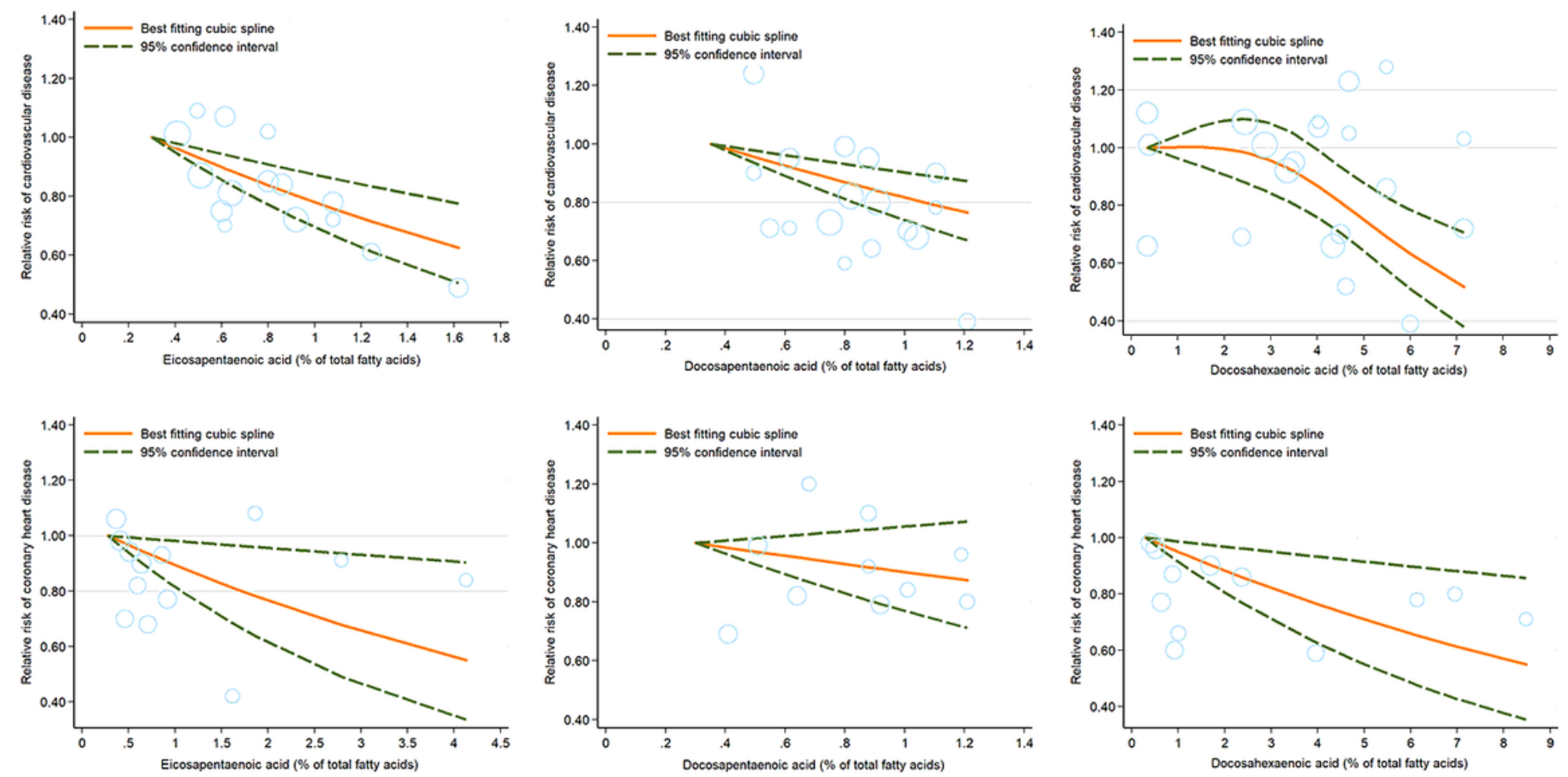

\section{Figure 3}

Dose-response analysis for linear or nonlinear association of EPA, DPA, and DHA biomarkers with CVD (A, $B$, and $C)$ and $C H D(D, E$, and $F)$ risk. The size of the black squares indicates the relative weight of each estimate, horizontal lines indicate $95 \%$ confidence interval $(\mathrm{Cl})$, and diamonds indicate summary relative risk (RR) estimates with 95\% Cls. CHD, coronary heart disease; CVD, cardiovascular disease; DHA, docosahexaenoic acid; DPA, docosapentaenoic acid; EPA, eicosapentaenoic acid; T2D, type 2 diabetes.

\section{Supplementary Files}

This is a list of supplementary files associated with this preprint. Click to download.

- Additionalfile1.docx

- Additionalfile2.docx

- Additionalfile3.docx

- Additionalfile4.docx

- Additionalfile5.docx

- Additionalfile6.docx

- Additionalfile7.docx 